\title{
LIDERADO TRANSFORMADOR, ENGAGEMENT E CREATIVIDADE NO CONTEXTO DE PEMES INTENSIVAS EN COÑECEMENTO
}

\author{
Guadalupe VILA VÁZQUEZ < guadalupe.vila@usc.es> \\ Carmen CASTRO CASAL <carmela.castro.casal@usc.es> \\ Dolores ÁLVAREZ PÉREZ mdolores.alvarez@usc.es \\ Universidade de Santiago de Compostela
}

RESUMO: A creatividade individual constitúe un input fundamental para a innovación e a competitividade das pemes intensivas en tecnoloxía e coñecemento. Este artigo considera que os supervisores cun estilo de liderado transformador poden estimular a creatividade dos empregados mediante o engagement. Os resultados do estudo, contrastado mediante ecuacións estruturais nunha mostra de empregados de pemes intensivas en tecnoloxía e coñecemento, así o confirman. Este achado supón importantes implicacións para a dirección das pemes intensivas en tecnoloxía e coñecemento que se enfronta ao desafío de potenciar a creatividade dos empregados.

PALABRAS CHAVE: Liderado transformador, engagement, creatividade, pemes intensivas en tecnoloxía e coñecemento.

ABSTRACT: Individual creativity is a fundamental input for the innovation and competitiveness of high-tech and knowledge based SMEs. This article argues that the supervisor's transformational leadership behavior can stimulate employees' creativity through engagement. This was confirmed by results of the study, tested using a structural equation modelling on a sample of employees from high-tech and knowledge based SMEs. This finding has important implications for the management of high-tech and knowledge based SMEs facing the challenge of enhancing the creativity of their employees.

KEYWORDS: Transformational leadership, engagement, creativity, high-tech and knowledge based SMEs.

\section{INTRODUCIÓN}

Na economía baseada no coñecemento, as empresas son máis dependentes da creatividade dos empregados (Anderson, Potočnik e Zhou, 2014; Shin e Zhou, 2003). A creatividade fai referencia á xeración de ideas novas e útiles para a empresa (Amabile et al., 1996). As pemes que operan en sectores intensivos en tecnoloxía e coñecemento precisan que os empregados xeren novas ideas e exploren solucións non convencionais (Rauch e Hatak, 2016) como base para a innovación e a introdución de novos procesos e produtos no mercado (Binyamin e Carmeli, 2010).

Son os empregados os que crean, desenvolven e aplican as novas ideas, proporcionando os recursos para a innovación (Bommer e Jalajas, 2002). Crear un contexto que apoie á creatividade e mobilice aos recursos humanos (Greer, Carr e Hipp, 2016) é, polo tanto, un dos principais desafíos aos que se enfrontan estas empresas.

O liderado transformador constitúe un factor da contorna de traballo que pode fomentar a creatividade e a innovación dos empregados (Amabile, 1997; Anderson et al., 2014; Shin e Zhou, 2003). Os líderes que se comportan de forma transformadora inspiran aos seguidores para que vaian máis aló dos seus intereses particulares e os motivan a facer máis do que inicialmente crían posible (Bass, 1990).

A literatura do liderado analizou extensamente a influencia do liderado nas actitudes, as cognicións e os comportamentos dos empregados (véxanse as revisións meta-analíticas de Judge e Piccolo, 2004 e Wang et al., 2011). Asemade, sinalouse que a relación entre o liderado transformador e os comportamentos individuais, entre eles a creatividade, é complexa. Neste artigo considérase que o engagement dos empregados 
constitúe un vínculo entre o liderado transformador do supervisor e a creatividade dos empregados no contexto de pemes novas intensivas en tecnoloxía e coñecemento.

Esta investigación contribúe á literatura actual de varias formas. Primeiro, malia que o liderado transformador pode predicir varias formas de motivación, como o engagement, conforme á revisión de Hiller et al. (2011), só 36 artigos publicados en revistas de alto impacto no período 1985-2009 analizaron directamente o efecto do liderado nos resultados motivacionais.

Segundo, cobre una escaseza de investigación sobre o efecto do engagement na creatividade dos empregados. Só dous estudos analizaron esta relación (Bakker e Xanthopoulou, 2013; Eldor e Harpaz, 2016) e ningún deles no contexto das pemes intensivas en tecnoloxía e coñecemento nas que a creatividade é un input fundamental para a innovación e a competitividade (Binyamin e Carmeli, 2010).

Terceiro, a evidencia de resultados inconsistentes nalgúns estudos sobre a relación liderado transformador-creatividade individual esixe investigar posibles mecanismos mediadores que permitan entender mellor o efecto do liderado transformador na creatividade (Anderson et al. 2014; Wang et al., 2011).

0 traballo estrutúrase como segue. Na primeira sección desenvólvese o marco conceptual que guía a investigación e formúlanse as hipóteses. Despois, exponse a metodoloxía seguida para a súa contrastación. Finalmente, discútense os resultados, sinálanse as limitacións do estudo e propóñense posibles ampliacións futuras.

\section{MARCO CONCEPTUAL}

\subsection{Liderado transformador e engagement}

0 engagement converteuse nun dos construtos fundamentais da investigación organizativa (Crawford et al., 2014; Saks e Gruman, 2014). Dende a perspectiva de Kahn (1990), o primeiro autor en definir o termo, o engagement é o emprego e a expresión física, emocional e cognitiva do auténtico eu no traballo. Constitúe pois un construto motivacional que describe o grao no que os empregados poñen de maneira simultánea todas as súas enerxías no desempeño dos seus roles de traballo (Rich, Lepine e Crawford, 2010).

Conforme á Kahn (1990), as percepcións dos empregados sobre o contexto de traballo e as súas características individuais promoven os estados psicolóxicos que determinan o grao no que decidirán dirixir todas as súas enerxías cara o rol laboral.

A literatura destaca o maior efecto do liderado transformador fronte ao transaccional no engagement dos seguidores. Os líderes transaccionais motivan aos seguidores para que realicen o traballo e recompénsanos por iso. Este estilo de liderado ten menos poder motivacional (Breevaart et al., 2014) e carece do atractivo inspirador do liderado transformador (Tims, Bakker e Xanthopoulou, 2011).

Malia que algúns estudos recentes analizaron a influencia do liderado transformador no engagement dos seguidores (p. ex., Aryee et al., 2012; Kopperud, Martinsen e Humborstad, 2014), a evidencia empírica aínda é escasa (Crawford et al., 2014, Saks e Gruman, 2014). Como destacan Carasco-Saul, Kim e Kim (2015, p. 38): “O liderado é un dos tópicos máis estudados nas ciencias organizativas e o engagement dos empregados un dos máis recentes. Porén, a relación entre o liderado e o engagement non foi amplamente investigada".

De acordo coa teoría de Kahn (1990), os líderes poden xerar engagement nos seguidores promovendo unha contorna na que experimenten máis seguridade psicolóxica, é dicir, "a sensación de poder amosar e empregar o seu auténtico eu sen temer sufrir consecuencias negativas" (Kahn, 1990, páx. 708). Algúns estudos (p. ex., Carmeli et al., 
2014) e revisións meta-analíticas (Frazier et al., 2017) corroboran que os líderes transformadores infunden nos seguidores sentimentos de seguridade psicolóxica.

Os líderes transformadores crean un contexto no que os seguidores se sintan animados a ser eles mesmos e a se expresaren libremente para desenvolver o seu potencial (Carmeli et al., 2014; May, Gilson e Harter, 2004). Ao estimular intelectualmente aos seguidores, animándoos a cuestionar os supostos establecidos e a enfocar os problemas de forma distinta (Bass e Riggio, 2006), transmiten a mensaxe de que se poden expresar abertamente, sen medo a consecuencias negativas (Carmeli et al., 2014; Kahn, 1990; Frazier et al., 2017).

Os líderes transformadores teñen en conta as necesidades de logro e desenvolvemento dos seguidores, proporciónanlles os recursos necesarios e establecen interaccións personalizadas con cada un (Bass, 1990). Como consecuencia, os seguidores experimentarán máis apoio e sentiranse psicoloxicamente máis seguros para introducir o seu eu no traballo (Kahn, 1990).

A seguridade psicolóxica, e con iso o desenvolvemento do engagement dos seguidores, baséase no grao de confianza e respecto que inspire o líder (Kahn 1990). Os líderes transformadores preocúpanse polos seguidores, apóianos e anímanos, e con iso gañan o seu respecto e confianza (Zhu et al., 2013). O estudo de May et al. (2004) amosou que o liderado de apoio do supervisor influíu positivamente no sentimento de seguridade psicolóxica dos seguidores e que este, asemade, fomentou o engagement. A meta-análise de Fraizer et al. (2017) revelou que a nivel individual, a seguridade psicolóxica está correlacionada co engagement.

Cando os seguidores consideran que os líderes constitúen modelos de rol axeitados -é dicir, cando antepoñen os intereses do grupo aos seus e están dispostos a realizar sacrificios persoais polo ben grupal (Bass e Riggio, 2006)-, confiarán máis neles, sentiranse máis orgullosos da organización, traballarán máis duro para acadar as metas (Zhu et al., 2013) e aumentarán o seu nivel de engagement (Vogelgesang, Leroy e Avolio, 2013).

Unha característica fundamental dos líderes transformadores é o senso de conexión que crean entre os seguidores (Kovjanic et al., 2012). Mediante o carisma e a dirección baseada no exemplo gañan o respecto e a admiración dos empregados. Iso, xunto coa consideración das necesidades particulares dos seguidores, fortalece o vencello líder-seguidor (Tse, Huang e Lam, 2013). Algunhas investigacións confirman que as relacións cos supervisores caracterizadas pola confianza e o apoio mutuos permiten que os empregados contribúan á organización de maneira construtiva cun sentimento de enerxía (Atwater e Carmeli, 2009) e engagement (Agarwal et al., 2012).

O liderado transformador tamén pode influír no engagement ao propiciar a percepción de significación e dispoñibilidade psicolóxicas (Crawford et al., 2014). Os empregados estarán dispostos a canalizar todas as súas enerxías cara a realización do seu rol se cren que contribúen a algo valioso e importante e que contan cos recursos persoais necesarios. Os líderes transformadores establecen e comunican expectativas de alto desempeño que desafían e inspiran aos empregados a acadar máis do que crían posible (Bass e Riggio, 2006). Coforme a Shamir, House e Arthur (1993), os líderes carismáticos melloran as expectativas de esforzo-logro dos seguidores ao vencellar o esforzo con valores importantes e ao amosar confianza nas súas habilidades para satisfacelas. Deste xeito, reforzan a significación do traballo e as crenzas de autoeficacia e valía persoal dos seguidores (Kark, Shamir e Chen, 2003; Tims et al., 2011).

Tomando como referencia a teoría da autodeterminación (Deci e Ryan, 2000), arguméntase que a probabilidade de que os empregados internalicen as tarefas e exhiban altos niveis de enerxía, concentración e persistencia aumenta se ven satisfeitas as súas 
necesidades básicas de autonomía, competencia e pertenza (Kovjanic, Schuh e Jonas, 2013). E os líderes transformadores proporcionan importantes recursos (autonomía, feedback, apoio, oportunidades de desenvolvemento, etc.) que contribúen a isto (Kovjanic et al., 2012; 2013).

Algúns estudos emprendidos en mostras de empregados doutros contextos corroboran o vencello directo entre o liderado transformador e o engagement: empregados dunha compañía de servizos financeiros e dunha auditora en Noruega (Kopperud et al., 2014), empregados dunha compañía de telecomunicacións en China (Aryee et al., 2012) e enxeñeiros e informáticos alemáns (Vincent-Höper, Muser e Janneck, 2012).

A partir dos argumentos previamente expostos, formúlase a seguinte hipótese:

Hipótese 1: O liderado transformador do supervisor inmediato influirá positivamente no engagement dos empregados.

\subsection{Engagement e creatividade}

Malia que Kahn (1990) non analizou explicitamente a influencia do engagement na creatividade, sinalou que os empregados engaged amosan a súa verdadeira identidade, pensamentos e sentimentos no traballo. E esta autoexpresión é a base da creatividade (Kahn, 1990).

A teoría dos tres compoñentes da creatividade (Amabile, 1997) formula que a creatividade, a nivel individual, depende de tres factores: pericia, habilidades de pensamento creativo e motivación intrínseca. A pericia inclúe os coñecementos, as habilidades técnicas e o talento no dominio do traballo. As habilidades de pensamento creativo comprenden un estilo cognitivo que permita enfocar os problemas dende diferentes perspectivas, a aplicación de técnicas heurísticas para explorar novas ideas e un estilo de traballo enérxico e persistente. A motivación intrínseca refírese ao desexo de se esforzar polo propio interese e desfrute co traballo que se realiza (Amabile et al., 1996; Deci e Ryan, 2000). Conforme a Amabile (1997), namentres que os dous primeros factores determinan a capacidade das persoas para se comporten de forma creativa, o terceiro é o que condiciona o seu comportamento.

Para Amabile (1997), a motivación intrínseca marca a diferenza entre o que unha persoa é capaz de facer nun determinado eido e o que realmente fará. Conforme á autora, "ata certo punto, unha alta motivación intrínseca pode chegar a compensar carencias na pericia ou nas habilidades de pensamento creativo" (Amabile, 1997, páx. 44). As persoas que están intrinsecamente motivadas tenden a ser curiosas e máis cognitivamente flexibles. Tamén adoitan asumir máis riscos e persistir perante as dificultades e desafíos que atopan. Todo iso facilita que desenvolvan ideas novas e útiles. Algúns estudos empíricos (p. ex., Shin e Zhou, 2003; Zhang e Bartol, 2010) corroboran que a creatividade dos empregados está influída pola motivación intrínseca.

0 engagement e a motivación intrínseca son construtos distintos, pero comparten semellanzas: ámbolos dous céntranse no esforzo e na perseveranza (Rich et al., 2010). Agora ben, o engagement no traballo é un construto motivacional máis amplo que abrangue o investimento simultáneo da enerxía física, emocional e cognitiva no rol laboral (Rich et al., 2010). Salanova e Schaufeli (2008) defenderon que o engagement cobre as dimensións básicas da motivación intrínseca, xa que favorece un comportamento orientado ás metas e persistente para acadar os obxectivos e caracterízase por altos niveis de activación e sentimentos de entusiasmo e identificación co traballo.

Baseándose nas premisas da teoría compoñencial da creatividade, Bakker e Xanthopoulou (2013) afirmaron que un empregado que non está engaged, non vai utilizar a súa pericia e as súas habilidades para ser creativo. Para Atwater e Carmeli (2009, p. 266) 
"sen enerxía, as habilidades creativas non se optimizarán e é menos probable que se utilicen creativamente no traballo". Pola contra, os empregados que se senten enérxicos, física e emocionalmente, e están concentrados no seu traballo utilizarán as súas destrezas e esforzaranse en maior medida por adquirir novas habilidades para seren creativos (Amabile, 1997).

Por outra banda, os estudosos do afecto e as emocións destacaron a importancia dos estados afectivos ${ }^{1}$ na creatividade. No modelo de ampliación e construción de emocións positivas (Fredrickson, 2001), a autora considera que ao experimentar emocións positivas, as persoas amplían o abano de pensamentos e accións que lles veñen á mente. Esta ampliación favorece o desenvolvemento de recursos intelectuais e psicolóxicos duradeiros que, á súa vez, promoven a exploración, a aprendizaxe e a creatividade.

Os estados afectivos positivos estimulan a creatividade porque amplían a gama de información cognitiva dispoñible, fan que as persoas estean máis atentas e asimilen un conxunto máis amplo de ideas, e identifiquen en maior medida patróns e conexións entre información e estímulos diferentes (Amabile et al., 2005; Fredrickson, 2001). Con iso, a probabilidade de que recoñezan os problemas, integren recursos diversos e sexan, como consecuencia, máis creativas aumenta.

Malia que algúns estudos de campo e laboratorio confirman a relación entre os estados afectivos positivos e a creatividade (p.ex.: Amabile et al., 2005), a evidencia é mixta (véxase Anderson et al., 2014).

Recentemente cuestionouse que todos os estados afectivos positivos estimulen de igual forma a creatividade. Conforme a Baas et al. (2008), é necesario ter en conta tamén o nivel de activación. Os estados de baixa activación levan á inacción e á evitación, a descoidar información e a un rendemento pobre en termos cognitivos. Os estados activadores, pola contra, melloran os procesos cognitivos, promoven a procura e integración de nova información e a identificación de múltiples alternativas.

A meta-análise levada a cabo por Baas et al. (2008) concluíu que os estados afectivos positivos altos en activación (felicidade, xúbilo) se asocian con maiores niveis de creatividade cós estados de ánimo neutrais. Porén, non ocorreu o mesmo cos estados de ánimo positivos e desactivadores (relaxamento, serenidade). Conforme aos autores, os estados de ánimo positivos e activadores favorecen a creatividade porque, ademais de incrementar a flexibilidade cognitiva, propician un procesamento máis eficiente e global. De Dreu, Baas e Nijstad (2008) constataron en varios estudos de laboratorio que os estados activadores e positivos aumentan a fluidez e a orixinalidade a través dunha maior flexibilidade cognitiva.

Os resultados doutras investigacións referendan que os estados afectivos activadores, como os sentimentos de enerxía, vitalidade e fortaleza física, relaciónanse positivamente coa creatividade. Nun estudo que abrangueu 193 empregados de distintos postos que traballaban en organizacións industriais e de servizo en Israel, Atwater e Carmeli (2009) evidenciaron que os empregados que sentían altos niveis de enerxía adoitaban involucrarse máis no traballo creativo que aqueles con niveis baixos. Dous estudos descubriron que a vitalidade (un estado afectivo positivo caracterizado por un alto nivel de activación, no que a persoa se sente chea de enerxía, viva e en pleno

\footnotetext{
${ }^{1} \mathrm{O}$ afecto é un termo xeral referido a un estado de sentimento subxectivo. Os estados de ánimo e as emocións son tipos de afecto. A diferenza dos estados de ánimo, as emocións están dirixidas cara un estímulo específico (unha persoa, un obxecto ou un evento), son máis intensas e menos duradeiras (Baas, De Dreu e Nijstad, 2008).
} 
funcionamento) influíu positivamente na implicación dos empregados no traballo creativo (Kark e Carmeli, 2009) e na creatividade (Cohen-Meitar, Carmeli e Waldman, 2009).

0 engagement é un estado afectivo positivo caracterizado por altos niveis de enerxía e activación. Así mesmo, o engagement denota como de intensa e persistentemente os empregados aplican as súas enerxías no rol laboral (Rich et al., 2010). A perseveranza e a persistencia cognitiva contribúen a xerar máis ideas e novas solucións (De Dreu et al., 2008). Consecuentemente, é máis probable que os empregados con alto engagement amosen máis curiosidade, estean dispostos a asumir máis riscos, procuren con entusiasmo novas ideas e exploren vías e enfoques non convencionais para solucionar os problemas e desafíos (Baer e Oldman, 2006; Chang et al., 2013).

Se ben algúns estudos analizaron o efecto do engagement no comportamento innovador dos empregados, a meirande parte en países asiáticos (p. ex., Agarwal et al., 2012; Aryee et al. 2012; Chang et al., 2013), a evidencia na creatividade é escasa. Malia que a creatividade e a innovación están relacionadas, non son sinónimos. A creatividade é unha condición previa do comportamento innovador (Anderson et al. 2014). Ata o momento, só dous estudos examinaron a influencia do engagement na creatividade. Bakker e Xanthopoulou (2013) amosaron que as directoras de escola engaged eran consideradas máis creativas polos profesores. Os resultados do recente estudo de Eldor e Harpaz (2016) revelan que o engagement fomentou a creatividade dos empregados de distintas angueiras e organizacións en Israel.

De acordo cos argumentos desenvolvidos, formúlase a seguinte hipótese:

Hipótese 2: 0 engagement influirá positivamente na creatividade dos empregados.

A teoría dos tres compoñentes da creatividade considera que o contexto de traballo (a orientación da organización cara a innovación, os recursos dispoñibles e as prácticas directivas como o apoio e o estímulo do supervisor) afecta á creatividade ao influír nos compoñentes individuais, pero o seu efecto é máis directo na motivación (Amabile, 1997). Eldor e Harpaz (2016) atoparon que o engagement individual mediu o efecto do clima de aprendizaxe (a nivel agregado) na creatividade individual. 0 estudo de Aryee et al. (2012) evidenciou que o engagement é un mediador da relación entre o liderado transformador e o comportamento innovador dos empregados.

Xa que se espera que o liderado transformador do supervisor impulse o engagement dos seguidores e que este, á súa vez, fomente a creatividade individual, proponse a seguinte hipótese:

Hipótese 3: 0 engagement mediará a relación entre o liderado transformador do supervisor e a creatividade dos empregados.

Na figura 1 plásmase o modelo conceptual.

Figura 1. Modelo formulado

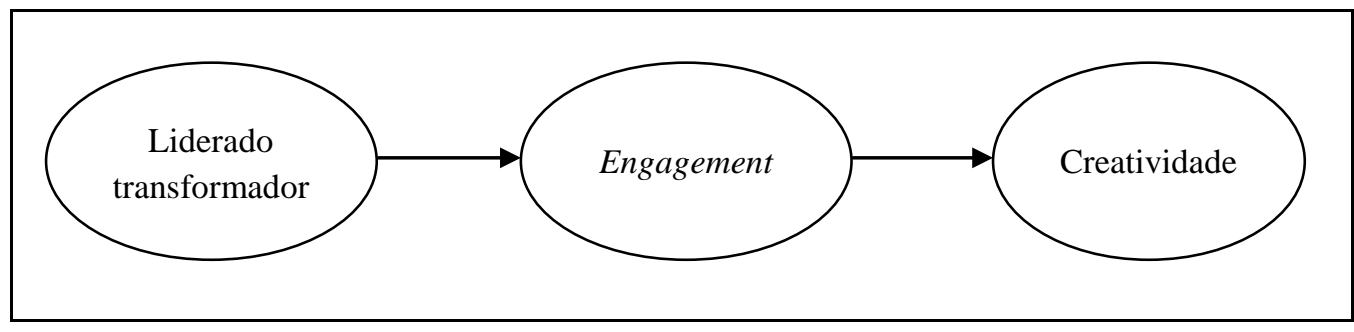




\section{METODOLOXÍA}

\subsection{Mostra}

Dado que o foco desta investigación son os empregados de pemes novas e intensivas en tecnoloxía e coñecemento, identificáronse 100 pemes, cunha idade máxima de 10 anos (Messersmith e Guthrie, 2010), que operaban nestes sectores conforme á clasificación utilizada por EUROSTAT. Delas, 21 colaboraron no estudo.

Estas pemes empregan entre 10 e 100 traballadores, e están situadas en diversas comunidades: Andalucía (3), Asturias (1), Castela-León (2), Cataluña (3), Extremadura (1), Galicia (6) e Madrid (5).

A distribución por sectores é a seguinte: Indústria química (2), fabricación de produtos informáticos, electrónicos e ópticos (1), programación, consultoría e outras actividades relacionadas coa informática (7), investigación e desenvolvemento (9) e outras actividades profesionais, científicas e técnicas (2).

As empresas participantes difundiron o interese do proxecto entre os empregados e proporcionaron ás autoras o e-mail de contacto dos empregados para distribuír a enquisa online. No e-mail enviado explicouse o interese do estudo e incorporouse un enlace á enquisa online, garantindo así o anonimato das respostas.

Recibíronse 320 cuestionarios válidos, o que representa unha taxa de resposta do $72,2 \%$. A mostra está conformada por un $63,75 \%$ de homes. A maioría dos empregados posúe un alto nivel de formación: o 69,06 \% ten estudos de educación superior e o $27,81 \%$ de máster ou doutoramento. A idade media dos empregados situouse en 32,09 anos.

Dado que a mostra inclúe empregados de pemes localizadas en distintas comunidades e pertencentes a distintos sectores de actividade, realizáronse ANOVAS para verificar se existían diferenzas nas medias das variables do estudo. Os resultados amosaron que as medias dos construtos dos diferentes grupos, por localización e sector, non son significativamente diferentes.

\subsection{Medidas e análise}

Todas as escalas foron previamente validadas e utilizadas en investigacións anteriores. Agás as variables de control, todas as variables medíronse con escalas tipo Likert de 7 puntos. As respostas flutuaban dende "moi en desacordo" (1) ata "moi de acordo" (7).

Liderado transformador. A percepción do liderado transformador do supervisor valorouse coa escala de Liderado Transformador Global de Carless, Wearing e Mann (2000). Esta escala, de carácter unidimensional, comprende 7 ítems que fan referencia aos seguintes comportamentos do líder: comunicar a visión, desenvolver ao persoal, proporcionar apoio, empoderar ao persoal, ser innovador, dirixir co exemplo e ser carismático. $0 \propto$ foi de 0,964 .

Engagement. Mediuse mediante a escala de Engagement no Posto de Rich et al. (2010), baseada no conceptualismo teórico de Kahn (1990). Esta escala considera o engagement como un construto latente de segunda orde formado por tres dimensións: engagement físico, engagement emocional e engagement cognitivo. En cada dimensión seleccionáronse os 4 ítems de maior carga factorial. Esta medida enfatiza a natureza motivacional do construto, polo que é especialmente indicada para analizar o efecto do engagement na creatividade dos empregados. $0 \alpha$ foi de 0,930 .

Creatividade. Este construto mediuse coa escala utilizada por Baer e Oldham (2006). $0 \propto$ foi de 0,909 .

Variables de control. Al igual que en investigacións previas (Agarwal et al., 2012; Aryee et al., 2012; Eldor e Harpaz, 2016), controláronse a idade, o xénero e o e nivel 
educativo dos empregados. A idade transformouse a Ln. Con respecto ao sexo, esta variable tomou o valor 0 para home e 1 para muller. 0 nivel de formación codificouse da seguinte forma: titulación de FP de grao medio $=1$; bacharelato $=2$; titulación de FP de grao superior $=3$; titulación universitaria $=4$; máster $=5$; e doutoramento $=6$.

A análise dos datos executouse en dous pasos. En primeiro lugar, efectuouse unha análise factorial confirmatoria para examinar o axuste do modelo de medida e valorar a consistencia interna de cada construto, a validez converxente das escalas e a validez discriminante dos construtos. En segundo lugar, estimouse o modelo estrutural. 0 modelo formulado comparouse con diversos modelos alternativos plausibles dende o punto de vista teórico.

As análises efectuáronse co software de ecuacións estruturais IBM SPSS Amos 21 para Windows, utilizando o método de estimación de máxima verosimilitude.

Para testar a significación do efecto indirecto utilizouse o procedemento non paramétrico bootstrapping (Hayes, 2009). Este método estatístico constrúe aleatoriamente un número de remostraxes da mostra orixinal coa finalidade de estimar os parámetros. No presente estudo obtívose un intervalo de confianza do efecto indirecto do $95 \%$ con 2000 remostraxes bootstrap.

\section{RESULTADOS}

O engagement maniféstase nas enerxías físicas, emocionais e cognitivas investidas de maneira simultánea no rol. Por iso, efectuouse unha análise factorial confirmatoria para avaliar a estrutura do construto como un factor de segunda orde. Especificouse un modelo no que as tres dimensións cargaban nun factor de orde superior. 0 axuste do modelo é satisfactorio $(\chi 2(49)=119,601 ; \quad \chi 2 / d f=2,441 ; \quad$ CFI $=0,979 ; \quad$ TLI $=0,972$; RMSEA $=0,067)$. As cargas factoriais de segunda orde das dimensións física, emocional e cognitiva do engagement $(0,879,0,690$ e 0,866 , respectivamente) son todas positivas, altas e estatisticamente significativas, dando apoio á estrutura do construto como factor de segunda orde.

Logo disto, contrastouse o axuste do modelo de medida. Os índices de axuste do modelo de medida, son axeitados $(\chi 2(223)=574,711 ; \quad \chi 2 / d f=2,577 ; \quad$ CFI $=0,951$; TLI = 0,944; RMSEA = 0,070).

En tódolos casos, o índice de fiabilidade composta (FC) e o AVE superan os niveis mínimos recomendados de 0,6 e 0,5, respectivamente (Hair et al., 2010), garantindo así a fiabilidade das escalas.

Con respecto á validez converxente, os parámetros lambda estandarizados son significativos e superiores ao limiar mínimo de 0,5 (Hair et al., 2010). Tamén se evidenciou a validez discriminante, xa que as correlacións entre as variables consideradas amosan intervalos de confianza que non inclúen o valor unitario e o seu valor ao cadrado non supera o AVE (Hair et al., 2010). No Anexo resúmense as propiedades psicométricas das escalas.

Os resultados do modelo estrutural indican que o modelo formulado ( $\mathrm{MH}$ ) (liderado transformador $\rightarrow$ engagement $\rightarrow$ creatividade) axústase ben aos datos $(\chi 2(287)=673,994 ; \chi 2 / \mathrm{df}=2,348 ; \mathrm{CFI}=0,946 ; \mathrm{TLI}=0,946 ; \mathrm{RMSEA}=0,065)$.

A partir da teoría existente, formuláronse dous modelos alternativos. Xa que algunha investigación previa amosou un vencello directo entre o liderado transformador e a creatividade (Jaiswal e Dhar, 2016; Shin e Zhou, 2003), no modelo 1 engadiuse ademais esta relación. 0 modelo 2 contemplou só as relacións directas do liderado transformador e o engagement coa creatividade. Para determinar o mellor modelo, seguindo as recomendacións de Hair et al. (2010), establecéronse as diferenzas na $\chi 2$ en función dos 
graos de liberdade. Como se pode observar na táboa 1, o axuste do modelo formulado $(\mathrm{MH})$ é significativamente mellor có dos outros modelos.

Táboa 1. Comparación de modelos

\begin{tabular}{|c|c|c|c|c|c|c|c|c|c|}
\hline Modelos & $x^{2}$ & $d f$ & $x^{2} / d f$ & TLI & CFI & RMSEA & $\Delta x^{2}$ & $\Delta d f$ & $p$ \\
\hline M1 & 671,486 & 286 & 2,348 & 0,939 & 0,946 & 0,065 & & & \\
\hline MH & 673,994 & 287 & 2,348 & 0,939 & 0,946 & 0,065 & MH-M1=2,508 & 1 & ns \\
\hline M2 & 700,220 & 287 & 2,440 & 0,935 & 0,942 & 0,067 & $\mathrm{M} 2-\mathrm{M} 1=28,734$ & 1 & $<0,001$ \\
\hline
\end{tabular}

Para comprobar o apoio ás hipóteses, analizáronse as betas estandarizadas e a significación asociada (véxase figura 2). A hipótese 1 formulaba que o liderado transformador influirá positivamente no engagement dos empregados. Os resultados $(\beta=0,319, \mathrm{p}<0,001)$ así o confirman. Tamén se apoia a hipótese 2 xa que o engagement inflúe positivamente na creatividade $(\beta=0,397, \mathrm{p}<0,001)$.

Figura 2. Resultados do modelo estrutural

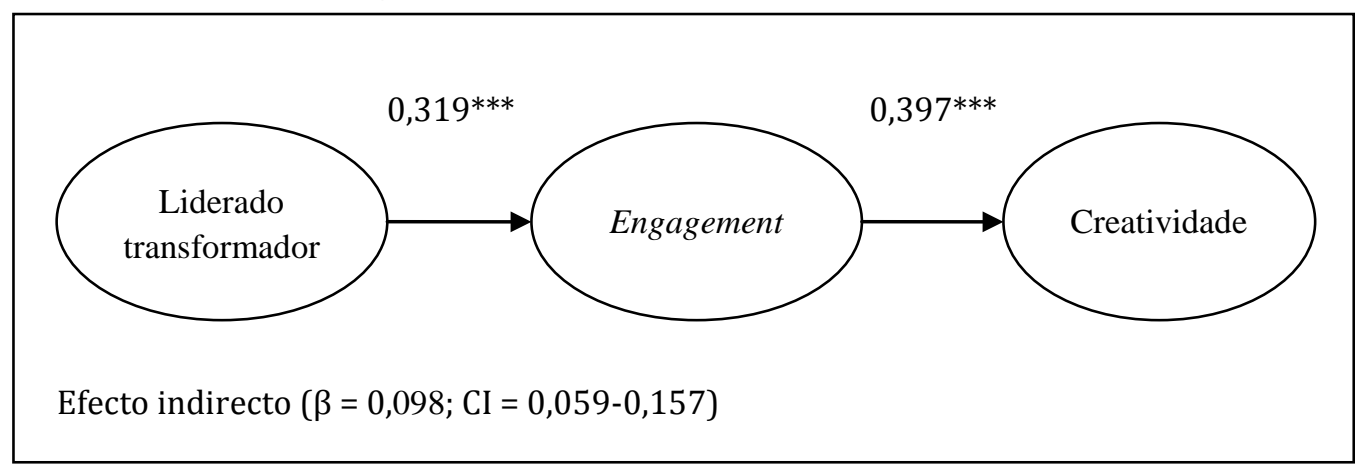

${ }^{* * *} \mathrm{p}<0,001$. Nota: Non se mostran os efectos das variables de control

A significación do efecto indirecto é positiva e significativa ao nivel $\mathrm{p}<0,001$, xa que os intervalos de confianza non inclúen o cero $(\beta=0,098 ; C I=0,059-0,157)$. Confírmase pois a hipótese 3: o liderado transformador do supervisor inflúe na creatividade dos empregados a través do engagement.

Con respecto ás variables de control, os resultados indican que os homes se consideran máis creativos cás mulleres. Namentres que o nivel educativo non inflúe na creatividade, a idade faino positivamente. Conforme a Ericsson (1999), a xeración de novas ideas pode aumentar coa idade como resultado da maior experiencia e pericia dos empregados. Estes resultados poden estar influídos polas características da mostra, conformada por empregados relativamente novos con alto nivel de formación. 


\section{DISCUSIÓN E CONCLUSIÓNS}

As pemes que operan en sectores intensivos en tecnoloxía e coñecemento deben fomentar a creatividade como base para a introdución de novos procesos e produtos no mercado. Estas empresas requeren empregados altamente cualificados e competentes, que estean dispostos a utilizar os seus coñecementos e habelencias para explorar novas ideas, solucións e oportunidades que lles permitan enfrontarse á contorna dinámica na que están inmersos.

Malia o recoñecemento de que os recursos humanos, a súa motivación e paixón son elementos críticos para a supervivencia e éxito das pemes (Pajo, Coetzer e Guenole, 2010), e, en particular das novas e pequenas empresas emprendedoras (Katz et al., 2000, p. 7), existe unha escaseza de investigación sobre como motivan aos empregados e fomentan a creatividade.

Os resultados da investigación previa sobre o efecto do liderado transformador na creatividade son conflitivos. Ao identificar o engagement como o nexo entre ámbalas dúas variables, este estudo permite entender mellor esta relación. Confírmase que o efecto do liderado transformador na creatividade dos empregados non é directo, senón que se produce vía engagement. O liderado transformador do supervisor propicia nos empregados un maior engagement e eles, á súa vez, alimentan un comportamento máis creativo.

Aínda que son os empregados os que deciden en que medida investirán o seu eu no rol, os supervisores crean as condicións axeitadas. Para Macey e Schneider (2008), os líderes transformadores son capaces de xerar nos seguidores sentimentos de paixón e identificación co traballo realizado. Os líderes transformadores axudan aos empregados a se desenvolveren, proporciónanlles o apoio e os recursos necesarios, fomentan a participación e a cooperación entre os membros do equipo e favorecen o pensamento crítico. Con estes comportamentos facilitan que os empregados poñan todas as súas enerxías no traballo.

En liña coa investigación sobre os estados afectivos positivos e de alta activación, os empregados que invisten as súas enerxías físicas, emocionais e cognitivas no rol tamén informan maior creatividade. Os empregados engaged experimentan emocións positivas, como interese, entusiasmo e enerxía (Rich et al., 2010), que lles levan a saír dos marcos de pensamento convencional, a experimentar e a xerar novas ideas e solucións diferentes (Eldor e Harpaz, 2016). Tamén é consistente cos estudos que destacan o papel da motivación intrínseca na creatividade (Shin e Zhou, 2003; Zhang e Bartol, 2010). Por analoxía, o engagement, un concepto motivacional máis amplo (Rich et al., 2010), asocíase coa creatividade.

Os resultados suxiren que o engagement é beneficioso para as pemes novas intensivas en tecnoloxía e coñecemento porque contribúe á creatividade dos empregados. Ningún dos estudos que examinaron empiricamente o vencello entre o engagement e a creatividade (Bakker e Xanthopoulou, 2013; Eldor e Harpaz, 2016) levouse a cabo nun contexto intensivo en coñecemento. Ao resolver esta carencia, esta investigación contribúe á literatura da creatividade.

Identificar o engagement dos empregados como o nexo entre o liderado transformador do supervisor e a creatividade dos seguidores permite comprender mellor os efectos positivos deste tipo de liderado nos empregados de pemes que operan en contornas dinámicas e se enfrontan a altas demandas de cambio. 0 liderado transformador do supervisor favorece a creación dunha contorna segura na que os empregados poidan experimentar e tratar coa ambigüidade característica destas empresas, permite aos empregados sentir unha maior significación no traballo e reafirma os seus sentimentos de valía persoal de tal forma que investirán o seu verdadeiro eu no 
rol. Arguméntase que os empregados engaged teñen probablemente unha concepción máis ampla do seu rol (Kahn, 1990; Rich et al., 2010). Estes empregados desempeñan o seu rol con entusiasmo e paixón, e poñen todas as súas enerxías nel, abranguendo todas as actividades que contribúen á súa efectividade (Rich et al., 2010).

Conforme a Shin e Zhou (2003), a diferenza das grandes empresas, as pemes novas non contan con regras e políticas formais que guíen o comportamento dos empregados; por iso, a súa dependencia do liderado do supervisor é maior. Supervisores e empregados traballan máis estreitamente unidos que nas grandes empresas. Para Mesu, Sanders e van Riemsdijk (2015), esta proximidade fai que o comportamento do supervisor afecte máis fortemente ás emocións e crenzas dos empregados que nas empresas de maior tamaño.

Malia que os investigadores comezaron a analizar posibles mecanismos na asociación liderado transformador-creatividade (p. ex., Shin e Zhou, 2003; Zhang e Bartol, 2010), "os procesos psicolóxicos subxacentes que relacionan o liderado transformador coa creatividade dos empregados non se entenden totalmente" (Henker, Sonnentag e Unger, 2015, p. 235). Polo tanto, o achado de que o engagement medía esta relación supón un importante avance que amplía a literatura existente.

Dende unha perspectiva práctica, as pemes novas e intensivas en coñecemento que desexen estimular a creatividade dos empregados deben prestar especial atención ao engagement. Diversas intervencións, como proporcionar aos empregados un traballo desafiante ou facer que se sintan apoiados pola organización, favorecen o engagement dos empregados (Kahn, 1990; Rich et al., 2010). Adicionalmente, os resultados deste estudo destacan o valor do liderado transformador.

Argumentouse que as prácticas de recrutamento e selección son determinantes do éxito ou fracaso das pequenas empresas (Greer et al., 2016) e que nas pemes e pequenas empresas innovadoras, elas se amplifican (Messersmith e Guthrie, 2010). Por iso, unha incorrecta selección terá quizabes consecuencias máis negativas nestas empresas que nas grandes e establecidas. Non é infrecuente que nas empresas tecnolóxicas o persoal directivo sexa seleccionado ou promocionado en función da súa experiencia e habilidades técnicas e préstese menos atención ás súas habilidades de liderado. A partir dos resultados achados, anímase á dirección a considerar as habilidades de liderado transformador na selección e promoción a postos directivos, e a comunicar as vantaxes deste tipo de liderado entre os supervisores actuais e potenciais.

Dado que o liderado é susceptible de desenvolvemento, é aconsellable formar aos supervisores e a aqueles que vaian ocupar algunha responsabilidade directiva en liderado transformador. Máis que un gasto, a formación en liderado transformador débese considerar un investimento: as empresas facilitarán que os empregados estean máis engaged, e sexan, como consecuencia, máis creativos. Asemade, estes programas deberíanse deseñar coidadosamente de forma que os custos non excedan os beneficios asociados.

O liderado transformador é particularmente axeitado nas pemes innovadoras ao incidir na motivación intrínseca fronte aos factores extrínsecos, máis característicos do liderado transaccional (Matzler et al., 2008). Adicionalmente, as restricións de recursos financeiros que adoitan afectar ás pemes, en comparación coas grandes empresas, dificultan ofertar altos incentivos monetarios para motivar o persoal.

Malia as implicacións comentadas, este estudo non está exento de limitacións. En primeiro lugar, a súa natureza transversal impide extraer conclusións sobre a dirección causal das relacións e descartar a posibilidade de causalidade inversa. É posible que os empregados máis engaged perciban que os supervisores lideran de forma máis transformadora ou que o maior engagement anime aos supervisores a adoptar este estilo. 
Malia que as relacións atopadas son consistentes en dirección e signo coa literatura, requírense estudos lonxitudinais ou experimentais que apoien a causalidade.

En segundo lugar, o feito de que todos os datos procedan da mesma fonte podería inflar as relacións como resultado da varianza do método común. Aínda que Spector (2006) suxeriu que o nesgo do método común rara vez é o suficientemente grave como para cuestionar a validez dos resultados, procurouse reducilo seguindo as recomendacións de Podsakoff, MacKenzie e Podsakoff (2012) relativas ao deseño do cuestionario. Separáronse as variables independentes das dependentes e garantiuse a confidencialidade e o anonimato dos participantes.

En terceiro lugar, o estudo centrouse nos empregados de pemes españolas novas intensivas en tecnoloxía e coñecemento. Malia a súa importancia para o crecemento económico e a innovación dun país (Desmarchelier, Djellal, e Gallouj, 2013), só unha pequena parte das pemes opera nestes sectores, o cal limita a xeneralización dos resultados. Investigacións futuras poderían tratar de replicar estes resultados en mostras de empregados de pemes pertencentes a outros sectores.

Mesmo coas limitacións, este estudo é o primeiro en explicar o proceso mediante o cal o liderado transformador do supervisor relaciónase coa creatividade dos empregados vía o engagement.

\section{BIBLIOGRAFÍA}

Agarwal, U. A.; Datta, S.; Blake-Beard, S.; Bhargava, S. (2012): "Linking LMX, innovative work behaviour and turnover intentions: The mediating role of work engagement", Career Development International, 17(3), pp. 208-230.

Amabile, T. M. (1997): "Motivating creativity in organizations: On doing what you love and loving what you do", California Management Review, 40(1), pp. 39-58.

Amabile, T. M.; Conti, R.; Coon, H.; Lazenby, J.; Herron, M. (1996): “Assesing the work environment for creativity". Academy of Management Journal, 39(5), pp. 1154-1184.

Amabile, T. M.; Barsade, S. G.; Mueller, J. S.; Staw, B. M. (2005): “Affect and creativity at work", Administrative Science Quarterly, 50(3), pp. 367-403.

Anderson, N.; Potočnik, K.; Zhou, J. (2014): “Innovation and creativity in organizations: A state-of-the-science review, prospective commentary, and guiding framework", Journal of Management, 40(5), pp. 1297-1333.

Aryee, S.; Walumbwa, F. O.; Zhou, Q.; Hartnell, C. A. (2012): “Transformational leadership, innovative behavior, and task performance: Test of mediation and moderation processes", Human Performance, 25(1), pp. 1-25.

Atwater, L.; Carmeli, A. (2009): "Leader-member exchange, feelings of energy, and involvement in creative work", The Leadership Quarterly, 20(3), pp. 264-275.

Baas, M.; De Dreu, C. K.; Nijstad, B. A. (2008): "A meta-analysis of 25 years of moodcreativity research: Hedonic tone, activation, or regulatory focus?", Psychological Bulletin, 134(6), pp. 779-806.

Baer, M.; Oldham, G. R. (2006): "The curvilinear relation between experienced creative time pressure and creativity: Moderating effects of openness to experience and support for creativity", Journal of Applied Psychology, 91(4), pp. 963-970.

Bakker, A. B.; Xanthopoulou, D. (2013): "Creativity and charisma among female leaders: The role of resources and work engagement", International Journal of Human Resource Management, 24(14), pp. 2760-2779.

Bass, B. M. (1990): "From transactional to transformational leadership: Learning to share the vision", Organizational Dynamics, 18(3), pp. 19-31.

Bass, B. M.; Riggio, R. E. (2006). Transformational leadership (2nd ed.). Mahwah, NJ: Lawrence Erlbaum Associates. 
Binyamin, G.; Carmeli, A. (2010): "Does structuring of human resource management processes enhance employee creativity. The mediating role of psychological availability", Human Resource Management, 49(6), pp. 999-1024.

Bommer, M.; Jalajas, D. (2002): "The innovation work environment of high-tech SMEs in the USA and Canada", R\&D Management, 32(5), pp. 379-386.

Breevaart, K.; Bakker, A. B.; Hetland, J.; Demerouti, E.; Olsen, O. K.; Espevik, R. (2014): "Daily transactional and transformational leadership and daily employee engagement", Journal of Occupational and Organizational Psychology, 87(1), pp.138-157.

Carasco-Saul, M.; Kim, W.; Kim, T. (2015): "Leadership and employee engagement proposing research agendas through a review of literature", Human Resource Development Review, 14(1), pp. 38-63.

Carless, S. A.; Wearing, A. J.; Mann, L. (2000): "A short measure of transformation leadership", Journal of Business \& Psychology, 14(3), pp. 389-406.

Carmeli, A.; Sheaffer, Z.; Binyamin, G.; Reiter-Palmon, R.; Shimoni, T. (2014): "Transformational leadership and creative problem-solving: The mediating role of psychological safety and reflexivity", The Journal of Creative Behavior, 48(2), pp. 115-135.

Chang, H.-T.; Hsu, H.-M.; Liou, J.-W.; Tsai, C.-T. (2013): "Psychological contracts and innovative behavior: A moderated path analysis of work engagement and job resources", Journal of Applied Social Psychology, 43(10), pp. 2120-2135.

Cohen-Meitar, R.; Carmeli, A.; Waldman, D. A. (2009): "Linking meaningfulness in the workplace to employee creativity: The intervening role of organizational identification and positive psychological experiences", Creativity Research Journal, 21(4), pp. 361-375.

Crawford, E. R.; Rich, B. L.; Buckman, B.; Bergeron, J. (2014): “The antecedents and drivers of employee engagement", en C. Truss, R. Delbridge, K. Alfes, A. Shantz y E. Soane (ed.): Employee Engagement in Theory and Practice, pp. 57-81, London and New York: Routledge.

Deci, E. L.; Ryan, R. M. (2000): "The" what" and" why" of goal pursuits: Human needs and the self-determination of behavior", Psychological Inquiry, 11(4), pp. 227-268.

De Dreu, C. K.; Baas, M.; Nijstad, B. A. (2008): "Hedonic tone and activation level in the mood-creativity link: Toward a dual pathway to creativity model", Journal of Personality and Social Psychology, 94(5), pp. 739-756.

Desmarchelier, B.; Djellal, F.; Gallouj, F. (2013): "Knowledge intensive business services and long term growth", Structural Change and Economic Dynamics, 25, pp. 188-205.

Eldor, L.; Harpaz, I. (2016): "A process model of employee engagement: The learning climate and its relationship with extra-role performance behaviors", Journal of Organizational Behavior, 37(2), pp. 213-235.

Ericsson, K. A. (1999): “Creative expertise as superior reproducible performance: Innovative and flexible aspects of expert performance", Psychological Inquiry, 10(4), pp. 329-361.

Frazier, M. L.; Fainshmidt, S.; Klinger, R. L.; Pezeshkan, A.; Vracheva, V. (2017): "Psychological safety: A meta-analytic review and extension", Personnel Psychology, 70(1), pp. 113165.

Fredrickson, B. L. (2001): "The role of positive emotions in positive psychology: The broaden-and-build theory of positive emotions", American Psychologist, 56(3), pp. 218-226.

Greer, C. R.; Carr, J. C.; Hipp, L. (2016): "Strategic staffing and small-firm performance", Human Resource Management, 55(4), pp. 741-764.

Hair, J. F.; Black, W. C.; Babin, B. J.; Anderson, R. E. (2010): Multivariate data analysis.7th ed. Prentice Hall, Englewood Cliffs.

Hayes, A. F. (2009): "Beyond Baron and Kenny: Statistical mediation analysis in the new millennium", Communication Monographs, 76(4), pp. 408-420.

Henker, N.; Sonnentag, S.; Unger, D. (2015): "Transformational leadership and employee creativity: The mediating role of promotion focus and creative process engagement", Journal of Business and Psychology, 30(2), pp. 235-247. 
Hiller, N. J.; DeChurch, L. A.; Murase, T.; Doty, D. (2011): "Searching for outcomes of leadership: A 25-year review", Journal of Management, 37(4), pp. 1137-1177.

Jaiswal, N. K.; Dhar, R. L. (2016): "Fostering employee creativity through transformational leadership: Moderating role of creative self-efficacy", Creativity Research Journal, 28(3), pp. 367-371.

Judge, T. A.; Piccolo, R. F. (2004): "Transformational and transactional leadership: A metaanalytic test of their relative validity", Journal of Applied Psychology, 89(5), pp. 755-768.

Kahn, W. A. (1990): "Psychological conditions of personal engagement and disengagement at work", Academy of Management Journal, 33(4), pp. 692-724.

Kark, R.; Carmeli, A. (2009): "Alive and creating: the mediating role of vitality and aliveness in the relationship between psychological safety and creative work involvement", Journal of Organizational Behavior, 30(6), pp. 785-804.

Kark, R.; Shamir, B.; Chen, G. (2003): "The two faces of transformational leadership: empowerment and dependency", Journal of Applied Psychology, 88(2), pp. 246-255.

Katz, J.; Aldrich, H.; Welbourne, T. M.; Williams, P. M. (2000): “Guest editor's comments special issue on human resource management and the SME: Toward a new synthesis", Entrepreneurship Theory and Practice, 25(1), pp. 7-10.

Kopperud, K. H.; Martinsen, Ø.; Humborstad, S. I. W. (2014): “Engaging leaders in the eyes of the beholder on the relationship between transformational leadership, work engagement, service climate, and self-other agreement", Journal of Leadership \& Organizational Studies, 21(1), pp. 29-42.

Kovjanic, S.; Schuh, S. C.; Jonas, K. (2013): “Transformational leadership and performance: An experimental investigation of the mediating effects of basic needs satisfaction and work engagement", Journal of Occupational \& Organizational Psychology, 86(4), pp. 543-555.

Kovjanic, S.; Schuh, S. C.; Jonas, K.; Quaquebeke, N. V.; van Dick, R. (2012): "How do transformational leaders foster positive employee outcomes? A self-determination-based analysis of employees' needs as mediating links", Journal of Organizational Behavior, 33(8), pp. 1031-1052.

Macey, W. H.; Schneider, B. (2008): “The meaning of employee engagement", Industrial and Organizational Psychology, 1(1), pp. 3-30.

Matzler, K.; Schwarz, E.; Deutinger, N.; Harms, R. (2008): “The Relationship between transformational leadership, product innovation and performance in SMEs", Journal of Small Business and Entrepreneurship, 21(2), pp. 139-151,251.

May, D. R.; Gilson, R. L.; Harter, L. M. (2004): "The psychological conditions of meaningfulness, safety and availability and the engagement of the human spirit at work", Journal of Occupational and Organizational Psychology, 77(1), pp. 11-37.

Messersmith, J. G.; Guthrie, J. P. (2010): "High performance work systems in emergent organizations: Implications for firm performance", Human Resource Management, 49(2), pp. 241264.

Mesu, J.; Sanders, K.; van Riemsdijk, M. (2015): “Transformational leadership and organisational commitment in manufacturing and service small to medium-sized enterprises: The moderating effects of directive and participative leadership", Personnel Review, 44(6), pp. 970-990.

Pajo, K.; Coetzer, A.; Guenole, N. (2010): "Formal development opportunities and withdrawal behaviors by employees in small and medium-sized enterprises", Journal of Small Business Management, 48(3), pp. 281-301.

Podsakoff, P. M.; MacKenzie, S. B.; Podsakoff, N. P. (2012): "Sources of method bias in social science research and recommendations on how to control it", Annual Review of Psychology, 63(1), pp. 539-569.

Rauch, A.; Hatak, I. (2016): “A meta-analysis of different HR-enhancing practices and performance of small and medium sized firms", Journal of Business Venturing, 31(5), pp. 485-504.

Rich, B. L.; Lepine, J. A.; Crawford, E. R. (2010): “Job engagement: Antecedents and effects on job performance", Academy of Management Journal, 53(3), pp. 617-635.

Saks, A. M.; Gruman, J. A. (2014): “What do we really know about employee engagement?", Human Resource Development Quarterly, 25(2), pp. 155-182. 
Salanova, M.; Schaufeli, W. B. (2008): "A cross-national study of work engagement as a mediator between job resources and proactive behavior", International Journal of Human Resource Management, 19(1), pp. 116-131.

Shamir, B.; House, R. J.; Arthur, M. B. (1993): "The motivational effects of charismatic leadership: A self-concept based theory", Organization Science, 4(4), pp. 577-594.

Shin, S. J.; Zhou, J. (2003): "Transformational leadership, conservation, and creativity: Evidence from Korea", Academy of Management Journal, 46(6), pp. 703-714.

Spector, P. E. (2006): "Method variance in organizational research truth or urban legend?", Organizational Research Methods, 9(2), pp. 221-232.

Tims, M.; Bakker, A. B.; Xanthopoulou, D. (2011): “Do transformational leaders enhance their followers' daily work engagement?", The Leadership Quarterly, 22(1), pp. 121-131.

Tse, H.; Huang, X.; Lam, W. (2013): "Why does transformational leadership matter for employee turnover? A multi-foci social exchange perspective", Leadership Quarterly, 24(5), pp. 763776.

Vincent-Höper, S.; Muser, C.; Janneck, M. (2012): "Transformational leadership, work engagement, and occupational success", Career Development International, 17(7), pp. 663-682.

Vogelgesang, G. R.; Leroy, H.; Avolio, B. J. (2013): "The mediating effects of leader integrity with transparency in communication and work engagement/performance", The Leadership Quarterly, 24(3), pp. 405-413.

Wang, G.; Oh, I.-S.; Courtright, S. H.; Colbert, A. E. (2011): "Transformational leadership and performance across criteria and levels: A meta-analytic review of 25 years of research", Group \& Organization Management, 36(2), pp. 223-270.

Zhang, X.; Bartol, K. M. (2010): “Linking empowering leadership and employee creativity: The influence of psychological empowerment, intrinsic motivation, and creative process engagement", Academy of Management Journal, 53(1), pp. 107-128.

Zhu, W.; Newman, A.; Miao, Q.; Hooke, A. (2013): "Revisiting the mediating role of trust in transformational leadership effects: Do different types of trust make a difference?", The Leadership Quarterly, 24(1), pp. 94-105.

\section{ANEXO. Propiedades psicométricas das escalas}

\begin{tabular}{|l|l|}
\hline Construtos & $\begin{array}{l}\text { Lambda estandarizado } \\
(\lambda)^{*}\end{array}$ \\
\hline Liderado transformador (AVE = 0,792; FC = 0,963) & 0,829 \\
\hline O meu supervisor comunica unha visión clara e positiva do futuro. & 0,937 \\
\hline $\begin{array}{l}\text { O meu supervisor trata ao persoal como individuos, apoia e fomenta o } \\
\text { seu desenvolvemento. }\end{array}$ & 0,928 \\
\hline O meu supervisor dá folgos e recoñecemento ao persoal. & 0,934 \\
\hline $\begin{array}{l}\text { O meu supervisor fomenta a confianza, a participación e a cooperación } \\
\text { entre os membros do equipo. }\end{array}$ & 0,811 \\
\hline $\begin{array}{l}\text { O meu supervisor fomenta pensar nos problemas de novas formas e } \\
\text { cuestiona os supostos establecidos. }\end{array}$ & 0,877 \\
\hline $\begin{array}{l}\text { O meu supervisor é claro en canto aos seus valores e practica o que } \\
\text { predica. }\end{array}$ & 0,906 \\
\hline $\begin{array}{l}\text { O meu supervisor infunde orgullo e respecto noutros e inspírame a ser } \\
\text { altamente competente. }\end{array}$ & \\
\hline
\end{tabular}




\begin{tabular}{|l|l|}
\hline Engagement (AVE $=\mathbf{0 , 6 6 4 ;} \mathbf{F C}=\mathbf{0 , 8 5 5 )}$ & \\
\hline Exerzo o meu esforzo completo no meu posto de traballo. & 0,827 \\
\hline Adico unha gran cantidade de enerxía ao meu posto de traballo. & 0,778 \\
\hline Esfórzome ao máximo para desempeñar ben ao meu posto de traballo. & 0,941 \\
\hline $\begin{array}{l}\text { Esfórzome todo o que podo para completar as tarefas do meu posto de } \\
\text { traballo. }\end{array}$ & 0,910 \\
\hline Son entusiasta no meu posto de traballo. & 0,914 \\
\hline Síntome enérxico no meu posto de traballo. & 0,866 \\
\hline Estou interesado no meu posto de traballo. & 0,702 \\
\hline Síntome optimista con respecto ao meu posto de traballo. & 0,727 \\
\hline No traballo, a miña mente está centrada no meu posto de traballo. & 0,872 \\
\hline No traballo, presto moita atención ao meu posto de traballo. & 0,963 \\
\hline $\begin{array}{l}\text { No traballo, centro una gran cantidad de atención no meu posto de } \\
\text { traballo. }\end{array}$ & 0,933 \\
\hline No traballo, estou absorto polo meu posto de traballo. & 0,630 \\
\hline Creatividade (AVE = 0,704; FC = 0,904) & 0,794 \\
\hline $\begin{array}{l}\text { Suxiro moitas ideas creativas que poderían mellorar as condicións de } \\
\text { traballo na organización. }\end{array}$ & 0,815 \\
\hline Concibo solucións creativas aos problemas no traballo. & 0,852 \\
\hline Suxiro novas formas de desempeñar as tarefas do traballo. & \\
\hline Son unha boa fonte de ideas creativas. & 0,892 \\
\hline *p < 0,001 & \\
\hline
\end{tabular}

\begin{tabular}{|c|c|c|c|}
\hline \multicolumn{4}{|l|}{ Validez discriminante: AVE e correlación ao cadrado das variables } \\
\hline & Liderado transformador & Engagement & Creatividade \\
\hline Liderado transformador & $\mathrm{AVE}=0,792$ & & \\
\hline Engagement & $\begin{array}{c}0,106 \\
{[0,209 ; 0,445]}\end{array}$ & $\mathrm{AVE}=0,664$ & \\
\hline Creatividade & $\begin{array}{c}0,000 \\
{[-0,095 ; 0,149]}\end{array}$ & $\begin{array}{c}0,155 \\
{[0,272 ; 0,516]}\end{array}$ & AVE $=0,704$ \\
\hline
\end{tabular}

Revista Galega de Economía: http://www.usc.es/econo/RGE/benvidag.htm 